\title{
Leishmaniose visceral canina em Maricá, Estado do Rio de Janeiro: relato do primeiro caso autóctone
}

\author{
Canine visceral leishmaniasis in Maricá, State of Rio de Janeiro: \\ first report of an autochthonous case
}

\author{
Cíntia Cristiane de Paula1, Fabiano Borges Figueiredo², Rodrigo Caldas Menezes², \\ Eliame Mouta-Confort ${ }^{1}$, Alessandra Bogio ${ }^{1}$ e Maria de Fátima Madeira ${ }^{1}$
}

\begin{abstract}
RESUMO
Leishmaniose visceral é uma zoonose de importância em Saúde Pública, onde os cães representam um dos maiores problemas. Este trabalho visa relatar o primeiro caso autóctone da leishmaniose visceral canina no município de Maricá, fornecendo elementos relacionados à distribuição geográfica de Leishmania (Leishmania) chagasi no Estado do Rio de Janeiro.
\end{abstract}

Palavras-chaves: Leishmaniose visceral. Leishmania chagasi. Cão. Maricá.

\begin{abstract}
Visceral leishmaniasis is a zoonosis of public health importance, and dogs represent one of the main problems. This paper describes the first autochthonous case of canine visceral leishmaniasis in the municipality of Maricá. It provides new facts regarding the geographical distribution of Leishmania (Leishmania) chagasi in the State of Rio de Janeiro.
\end{abstract}

Key-words: Visceral leishmaniasis. Leishmania chagasi. Dog. Maricá

A leishmaniose visceral (LV) é uma zoonose, de transmissão vetorial que tem como agente etiológico o protozoário Leishmania (Leishmania) chagasi. No Brasil, esta doença continua sendo um grande desafio nas questões de saúde pública, principalmente pelo potencial endêmico que vem assumindo em vários estados brasileiros $^{9}$. Cães são considerados os principais reservatórios domésticos, sendo monitorados por meio de inquéritos sorológicos, preconizados pelo Ministério da Saúde (MS), que visa a eliminação dos animais sororreatores?.

O Estado do Rio de Janeiro está classificado como área de transmissão esporádica para $\mathrm{LV}^{9}$ com casos caninos diagnosticados apenas na capital ${ }^{168}$, entretanto, recentemente foram relatados casos de leishmaniose visceral canina (LVC) nos municípios de Angra dos Reis e Mangaratiba, demonstrando a expansão da doença para outras regiões do estado ${ }^{6}$.

\footnotetext{
1. Laboratório de Vigilância em Leishmanioses, Instituto de Pesquisa Clínica Evandro Chagas, Fundação Oswaldo Cruz, Rio de Janeiro, RJ. 2. Laboratório de Pesquisa Clínica em Dermatozoonoses em animais domésticos, Instituto de Pesquisa Clínica Evandro Chagas, Fundação Oswaldo Cruz, Rio de Janeiro, RJ.

Apoio financeiro: Fundação de Apoio a Pesquisa do Estado do Rio de Janeiro(FAPERJ)

Endereço para correspondência: Dra. Cíntia Cristiane de Paula. Laboratório de Vigilância em Leishmanioses/IPEC/FIOCRUZ. Av. Brasil 4365, 21045-900 Rio de Janeiro, RJ.

Tel: 5521 3865-9541; Fax: 5521 3865-9541

e-mail: cintia.paula@ipec.fiocruz.br

Recebido para publicação em 04/09/2008

Aceito em 20/01/2009
}

O Município de Maricá está localizado na vertente litorânea do Estado do Rio de Janeiro (Latitude 22 ${ }^{\circ} 55^{\prime} 10^{\prime \prime}$ S Longitude $\left.42^{\circ} 49^{\prime} 07^{\prime \prime} 0\right)^{3}$, cuja característica geográfica proporciona atividades de veraneio favorecendo o processo de ocupação com severas consequiências para o meio ambiente ${ }^{10}$, fato associado ao aparecimento de casos de leishmaniose tegumentar americana (LTA) neste município ${ }^{71014}$.

Os autores visam relatar o primeiro caso autóctone de LVC no município de Maricá, Rio de Janeiro.

\section{RELATO DE CASO}

Um Cão de 7 anos de idade, proveniente do bairro de Inoã foi encaminhado ao Laboratório de Pesquisa Clínica em Dermatozoonoses em animais domésticos (Instituto de Pesquisa Clínica Evandro Chagas/Fundação Oswaldo Cruz) para confirmação diagnóstica, por suspeita de LVC. Segundo o proprietário, o cão havia nascido no local e possuía hábitos limitados ao quintal da residência sem nunca ter se ausentado. Ao exame clínico o animal apresentou emagrecimento, onicogrifose, adenite generalizada, esplenomegalia e pêlo opaco. Embora tais sinais sejam clássicos na LVC, podem ser confundidos com outras doenças, razão pela qual diferentes amostras biológicas foram coletadas para exames sorológicos e parasitológicos. Anticorpos IgG anti-Leishmania foram pesquisados utilizando kits de imunofluorescência indireta (IFI) e ensaio imunoenzimático (EIE) para LVC (Bio-Manguinhos/ FIOCRUZ/MS). Para o diagnóstico parasitológico, empregou-se 
o exame direto em aspirado de linfonodo poplíteo e cultivo de fragmentos de pele íntegra. A caracterização da amostra isolada, foi feita através da técnica de isoenzimas ${ }^{2}$, utilizando 7 sistemas enzimáticos.

0 resultado do exame direto evidenciou inúmeras formas amastigotas. Entretanto, foi o cultivo de fragmentos de pele que possibilitou o isolamento do parasita, identificado como Leishmania chagasi, comprovando tratar-se de um caso de IVC.

Todos os procedimentos foram aprovados pelo Comitê de Ética no Uso de Animais (CEUA-FIOCRUZ) sob o protocolo P.0298-06.

\section{DISCUSSÃo}

Em áreas urbanas, cães domésticos são considerados os principais reservatórios de Leishmania chagasi e embora a doença possa manifestar-se de maneira bastante variável, o parasitismo da pele constitui uma das características mais importantes, fazendo desse animal um elo no ciclo epidemiológico ${ }^{13}$. A retirada de cães sororreatores das áreas endêmicas, ainda é um assunto polêmico. Apesar de resultados sorológicos serem determinantes para eutanásia dos animais, em áreas de sobreposição com a forma tegumentar, tais resultados possuem valor limitado, já que não apresentam poder discriminatório para ambas as doenças, as quais possuem estratégias de controle diferenciadas para os cães infectados. 0 animal deste estudo apresentou reatividade tanto na IFI (1:80) como no EIE, no entanto, por tratar-se de uma área onde até então não havia registros da doença, a identificação etiológica foi fundamental para confirmação do caso, indicando a eutanásia do animal.

Os movimentos migratórios do homem com seus animais, associado às alterações ambientais são fatores importantes na disseminação geográfica de certas doenças ${ }^{11}$. Maricá é um município que enfrenta tais alterações, vinculadas principalmente às atividades de veraneio ${ }^{10}$, o que talvez possa ter favorecido a introdução da doença, entretanto não foram feitos maiores estudos nesse sentido.

Em várias regiões de Maricá, observam-se desmatamentos que dão lugar a construções humanas, como observado em Inoã ${ }^{710}$, local de residência do animal. Nesse contexto, o tipo de ocupação territorial foi associado ao surgimento de casos de LTA, verificado tanto em Maricá ${ }^{10}$, como no município do Rio de Janeiro ${ }^{4}$, tornando essas áreas vulneráveis à transmissão peridomiciliar de Leishmania braziliensis.

Um fato importante a ser destacado é a capacidade de domiciliação de Lutzomyia longipalpis ${ }^{5}$ em ambientes modificados. Souza e cols ${ }^{12}$ detectaram Lutzomyia intermedia em Inoã, no entanto, L. longipalpis ainda não foi descrita nessa localidade. A proximidade da residência do animal com a mata favorece o contato com animais sinantrópicos, que podem atuar como elo de transmissão de Leishmania chagasi ${ }^{1}$.
Os resultados apresentados demonstram pela primeira vez a presença de Leishmania chagasi em caso autóctone em Maricá e sinalizam a importância de estudos relacionados a fauna flebotomínica local, assim como a avaliação de outros cães, constituindo um alerta para vigilância epidemiológica, devendo ser considerada nas estratégias de controle da LVC.

\section{REFERÊNCIAS}

1. Cabrera MAA, Paula AA, Camacho LAB, Marzochi MCA, Xavier SC, Silva AVM, Jansen AM. Canine visceral leishmaniasis in Barra de Guaratiba, Rio de Janeiro, Brazil: assesssment of risk factors. Revista do Instituto de Medicina Tropical de São Paulo 45: 79-83, 2003.

2. Cupolillo E, Grimaldi Jr G, Momen H. A general classification of New World Leishmania using numerical zymotaxonomy. American Journal of Tropical Medicine and Hygiene 50: 296-311, 1994.

3. Governo do Estado do Rio de Janeiro. Anuário Estatístico, Texto Analítico. Disponível em: http://www.cide.rj.gov.br/Cidinho/pages/municipios.asp. Acessado em $07 / 08 / 2008$.

4. Kawa H, Sabroza PC. Espacialização da leishmaniose tegumentar na cidade do rio de janeiro. Caderno de Saúde Pública 18: 853-865, 2002.

5. Lainson R, Rangel EF. Lutzomyia longipalpis and the eco-epidemiology of american visceral leishmaniasis, with particular reference to Brazil - A review. Memórias do Instituto Oswaldo Cruz 100: 811-827, 2005.

6. Madeira MF, Schubach AO, Schubach TMP, Pereira AS, Figueiredo FB, Baptista C, Leal CA, Melo CX, Confort EM, Marzochi MC. Post mortem parasitological evaluation of dogs seroreactive for Leishmania from Rio de Janeiro, Brazil. Veterinary Parasitology 138: 366-370, 2006.

7. Madeira MF, Uchôa CMA, Leal CA, Silva RMM, Duarte R, Magalhães CM, Serra CMB. Leishmania (Viannia) braziliensis em cães naturalmente infectados. Revista da Sociedade Brasileira de Medicina Tropical 36: 551-555, 2003.

8. Marzochi MC, Coutinho SG, Souza WJ, Toledo LM, Grimaldi Jr G, Momen H, Pacheco RS, Sabroza PC, Souza MA, Rangel Jr FB, Tramontano NC. Canine visceral leishmaniasis in Rio de Janeiro, Brazil. Clinical, parasitological, therapeutical and epidemiological findings (1977-1983). Memórias do Instituto Oswaldo Cruz 80: 349-357, 1985.

9. Ministério da Saúde. Manual de vigilância e controle da Leishmaniose visceral. Ministério da Saúde, Brasília 122, 2006.

10. Serra CM, Leal CA, Figueiredo F, Schubach TM, Duarte R, Uchoa CM, Silva RM, Madeira MF. Canine tegumentary leishmaniasis in Morada das Águias (Serra da Tiririca), Maricá, Rio de Janeiro, Brazil. Cadernos de Saúde Pública 19: 18771880, 2003.

11. Shaw $\mathrm{J}$. The leishmaniases - survival and expansion in a changing world. A mini-review. Memórias do Instituto Oswaldo Cruz 102: 541-547, 2007.

12. Souza MB, Marzochi MCA, Leal CM, Ponte CS, Meródio JC, Meira AM, Pinha LCL. Leishmaniose tegumentar americana no município de Maricá- Rio de Janeiro. Estudo da fauna flebotomínica. Resumos do XXXVII Congresso da Sociedade Brasileira de Medicina Tropical, Salvador p. 221-222, 2001.

13. Travi BL, Tabares CJ, Cadena H, Ferro C, Osório Y. Canine visceral leishmaiasis in Colômbia: Relationship between clinical and parasitologic status and infectivity for sand flies. American Journal of Tropical Medicine and Hygiene 63: 119-124, 2001.

14. Uchôa CMA, Serra CMB, Duarte R, Magalhães CM, Silva RM, Theophilo F, Figliuolo LP, Horta FT, Madeira MF. Aspectos sorológicos da leishmaniose tegumentar americana canina em Maricá, Rio de Janeiro, Brasil. Revista da Sociedade Brasileira de Medicina Tropical 34: 563-568, 2001. 